\title{
Trayectoria y estrategias de emprendimiento en mujeres
}

Trajectory and

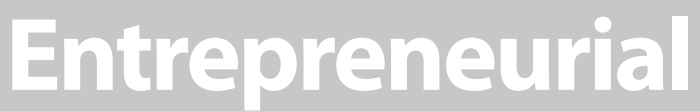

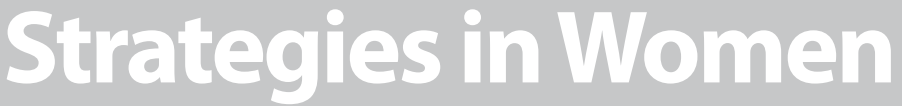

Dra. Ana Laura Arteaga Cervantes Profesor titular UPG aartega@upgto.edu.mx

Dr. José Felipe Ojeda Hidalgo Profesor titular UPG,

Profesor invitado CESBA

jojeda@upgto.edu.mx

Dra. Dolores Guadalupe Álvarez Orozco

Directora de vinculación, UPG

dalvarez@upgto.edu.mx
Arteaga Cervantes, A.L., Ojeda Hidalgo, J.F., y Álvarez Orozco, D.G., (2020)

Trayectoria y estrategias de emprendimiento en mujeres Revista Internacional de Investigación en Comunicación aDResearch ESIC. № 22 Vol 22

Monográfico especial, marzo 2020 · Págs. 176 a 195

https://doi.org/10.7263/adresic-022-10 
RESUMEN

Clasificación JEL:

L26, L1, J16

Palabras clave:

Emprendimiento, estrategias,

mujeres
Objetivo: El objetivo de la investigación será análisis las estrategias empleadas por mujeres dirigentes de empresas para mantener a su organización vigente en el mercado, dese la teoría de las cinco fuerzas de Porter, la teoría de los recursos y capacidades de Wernerfelt y la teoría de los capitales organizacionales

Diseño/metodología/enfoque: Para ello el método utilizado fue el cualitativo, a partir del paradigma interpretativo de la teoría fundamentada. Se entrevistaron a 40 mujeres dirigentes de empresas con una trayectoria que va de los 3 a los 56 años; en lugar de localizarlas se utilizó la técnica bola de nieve y las entrevistas fueron realizadas en sus centros de trabajo.

Resultados: Como principales hallazgos la investigación confirmó que las mujeres tienen una trayectoria que va de los 3 a los 56 años. De igual forma se identificó que emplean estrategias empíricas de manera inconsciente, en su gran mayoría no son formuladas a partir de una planeación o diseñadas con un objetivo específico, surgen de la atención a una necesidad de sus empresas, se generan y guían en la mayoría de los casos por la intuición.

Limitaciones/Implicaciones: Estos resultados hacen evidente la necesidad de estudiar la realidad latina, fuera de las ópticas anglosajonas, debido a la multiplicidad de estrategias que se utilizan en otras realidades y que no precisamente están reflejadas en la literatura más difundidas.

Originalidad/Contribución: Los resultados contradicen la teoría que propone la implementación de estrategias administrativas como medio de subsistencia de los negocios. En los casos estudiados se identificó que no es determinante y que existen excepciones exitosas las cuales manejan gestiones meramente intuitivas y han logrado subsistir y desarrollarse.

\section{ABSTRACT}

\section{JEL Classification:}

L26, L1, J16

Key words:

Entrepreneurship,

strategies,

women
Purpose: The objective of the research will be to analyze the strategies employed by women business leaders to keep their organization in force in the market, given the theory of Porter's five forces, the theory of resources and capabilities of Wernerfelt and the theory of structural capitals.

Design / Methodology / Approach: For this, the method used was the qualitative one, based on the interpretative paradigm of the grounded theory. 40 women business leaders were interviewed with a trajectory ranging from 3 to 56 years; to locate them the snowball technique was used and the interviews were conducted in their workplaces.

Results: The research confirmed that women have a trajectory ranging from 3 to 56 years. In the same way it was identified that they employ empirical strategies in an unconscious way and in the great majority they are not formulated from a planning or designed with a specific objective, they arise from attention to a need of their companies, they are generated and guided in the majority of cases by intuition.

Limitations / Implications: These results make the need to study the reality in Latin countries evident, outside of Anglo-Saxon optics, due to the multiplicity of strategies that are used in other realities and that are not precisely reflected in the most widespread literature.

Originality / Contribution:The results contradict the theory that proposes the implementation of administrative strategies as a means of subsistence of business. In the cases studied, it was identified that it is not decisive and that there are successful exceptions which handle merely intuitive efforts and have managed to survive and develop. 


\section{Introducción}

En México la población ocupada alcanzó 94,71 \% de la Población Económicamente Activa (PEA). Del total de ocupados, el 66,8 \% opera como trabajador subordinado y remunerado ocupando una plaza o puesto de trabajo, 4,2 \% son patrones o empleadores, 22,9 \% trabaja de manera independiente o por su cuenta sin contratar empleados y finalmente un 6,1\% se desempeña en los negocios o en las parcelas familiares, contribuyendo de manera directa a los procesos productivos, pero sin un acuerdo de remuneración monetaria (INEGI, 2019:4). Si el análisis de la población económicamente activa es relevante, lo es aún más cuando se identificar la participación en la generación de bienes económicos por género.

Estos datos como tal son trascendentales, pero toman mayor importancia si se complementan con estudios llevados a cabo por Encuesta $\mathrm{Na}$ cional de Ocupación y Empleo (ENOE) e INEGI y publicados por CNN Expansión, donde se establece que en los últimos años el $19 \%$ de los emprendedores en nuestro país son mujeres, lo que representa un considerable incremento en cuento al género en el número de emprendedores, destacando que hacia el año 1995 en el país había 2.5 millones de emprendedoras, cifra que se incrementó a 4 millones en el 2013 (El Economista America.com, 2019).

Al respecto existe discrepancia en cuanto al número de mujeres emprendedoras información de Maubert (2013) a la página de la Secretaria de economía cita lo siguiente:

La mexicana actual ya no sólo se encarga del hogar o su familia. Hoy representa más de una tercera parte de la población económicamente activa del país, y

\section{Figura 1. Población ocupada al segundo trimestre del 2019}

(Millones de personas)

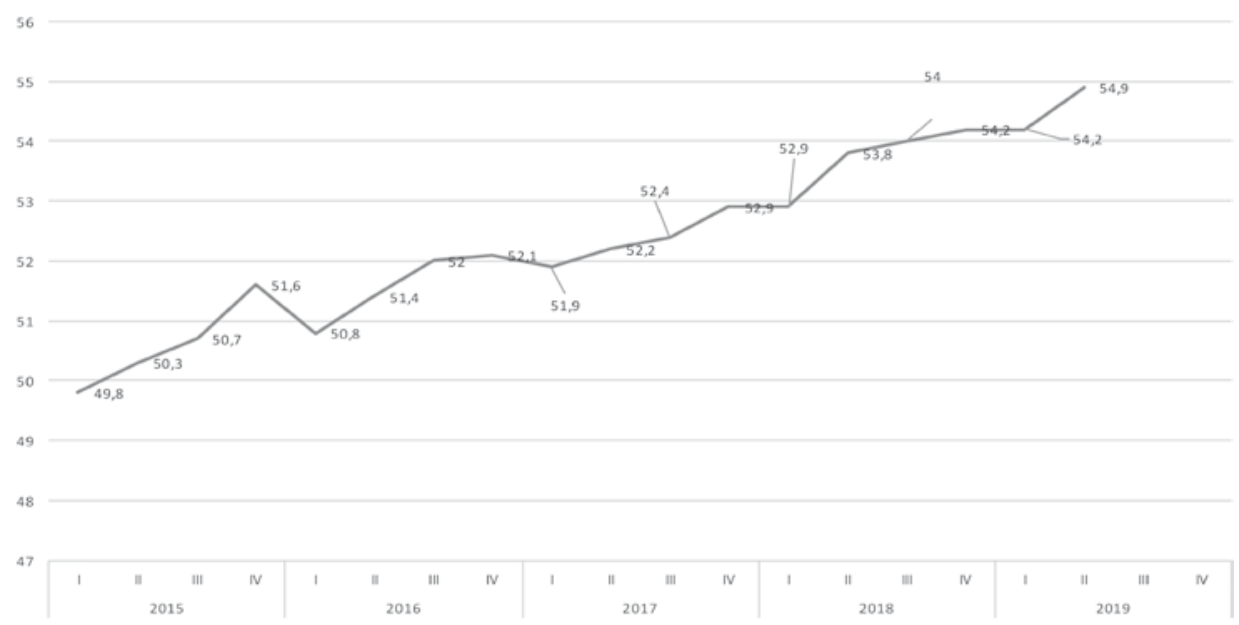


las que trabajan por su cuenta o tienen un negocio son cada vez más. Con un estilo de liderazgo flexible, cooperativo Y conciliador, las emprendedoras y ejecutivas enfrentan todavía muchos retos... pero están transformando sus empresas, sus mercados y nuestra manera de pensar (Maubert, 2013:1).

De lo anterior se genera la necesidad de formular una serie de preguntas tales como ¿cuál es la trayectoria que tienen las mujeres emprendedoras?, ¿Se pueden identificar estrategias empleadas por las mujeres emprendedoras? ¿Qué tipo de estrategias emplean las mujeres emprendedoras? Sin duda cada uno de los cuestionamientos realizados nos permitirá identificar las trayectorias y estrategias empleadas por mujeres que se han adentrado al mundo del emprendimiento, contribuyendo así al desarrollo económico tanto del país como de la economía de las familias.

\section{Revisión de la literatura}

\subsection{Emprendimiento}

Emprendimiento es considerado de origen reciente, sin embargo, si analizamos esta característica ha estado presente en la historia del ser humano, debido a que el emprendimiento puede ser considerado una capacidad de los seres humanos para salir adelante. Por lo que el ser emprendedor requiere tener habilidades tanto en lo individual como en lo colectivo. En los últimos años el termino ha tomado fuerza y se ha vuelto importante debido a la manera en cómo se ha manejado en términos económicos y cómo se han generado nuevas ideas (Jaramillo, 2008).

El concepto de emprendimiento se origina en Francia y tiene sinónimos como merhant, dealer, business person, entre otros, en los últimos años se ha entendido como un hombre o mujer, que tiene la capacidad de crear su propio negocio (Puchol, 2005).
A decir de Pereira (2007) el término entrepreneur aparece por vez primera en el texto Essai Sur la Nature du Commerce, en General esta palabra describe a una persona que se caracterizaba por comprar productos a precios conocidos para venderlos a precios desconocidos (Cantillon, 1755).

El emprendimiento puede ser visto como una de las características que llega a determinar el crecimiento, la transformación y el desarrollo de diversos sectores económicos de una región o un país, siendo el ser humano el principal motor para que esto se genere (Marulanda, Correa y Mejía, 2009).

Ramírez (2008) realiza un recorrido de la edad media al siglo XX resaltando las principales aportaciones del emprendimiento en cada uno de esos años. Para Hoselitz (1960), Say es uno de los grandes colaboradores del emprendimiento en este período, al manifestar que el empresario representaba o se constituía en el catalizador para el desarrollo de productos, y lo definía como un «trabajador superior». Consideraba que el fundamento del valor está en la utilidad que los distintos bienes reporten a las personas. Esta utilidad puede variar en función de la persona, del tiempo y del lugar en el contexto del emprendimiento. La concepción de Say es, por lo tanto, que el valor es subjetivo para el empresario (Rodríguez Ramírez, 2009:98).

A lo largo de 200 diversos autores han propuesto un concepto para entender el emprendimiento (Crisseien, 2009; Rodríguez 2009, Aurieu 2003; Filion, 2003; Bruinet y Alarcón, 2004, Bustos, 2005, Ekelund y Hernert, 2014, Formichella, 2004; Casco y Barrera, 2011; Quintero, 2007), a últimas fechas se considera que el emprendimiento es una acción inherente la potencial del desarrollo humano que responde a la necesidad de un sentido (Ensciso - Congote, 2008). 
El espíritu emprendedor se puede entender de varias formas, entre ellas a las personas empresarias, generadoras de beneficios para la sociedad (considerando la autorrealización, la generación y distribción de riquezas, generación de empleo, incremento en la dinámica empresarial, entre otros), integrando a su vez al emprendedor social, que es un generador de impacto en la propia sociedad (Leiva, 2007).

El emprendedor asume riesgos en condiciones de incertidumbre, dividiendo a los productores de la economía de mercado en concentrados, que reciben salarios o rentas fijas, y los emprendedores, que reciben ganancias variables e inciertas (Rodríguez, 2009).

El espíritu emprendedor puede constituirse en instrumento fundamental para favorecer la igualdad de oportunidades entre hombre y mujeres. En España las mujeres superan en número a los hombres licenciados universitarios, se cuenta con una tasa baja de empleo femenino en la Unión Europea. Por lo que el reto esta en lograr el aprovechamiento economico de los recurso humanos para lograr el equilibrio social integrando a las mujeres en un empleo (García, Moreno y Rosique, 2012).

García (2008) identifica tres escuelas del emprendimiento, la psicológica con las corrientes personalistas (Shaver y Scott, 1991), la escuela comportamental (Gartner, 1988) y el de la escuela económica; por otro lado Surdez, Aguilar y Rodríguez (2008) proponen las variables de liderazgo, propensión a asumir riesgos, creatividad / innovación, intuición, negociación, autoconfianza, necesidad de realización: ambición, trabajo arduo y audisciplina.

Estos emprendedores utilizan herramientas y procesos para sacar adelante el motivo del emprendimiento, es por ello que dentro del trabajo analizaremos el tema de la estrategia.

\subsection{Estrategia}

La estrategia puede ser entendida como un concepto tan viejo y antiguo como la guerra, es reconocida como «la habilidad, la pericia, para ubicar y mover al ejército en el campo de batalla con el fin no sólo de competir sino de ganar», se enlaza directamente con el ganar en todas y cada una de las actividades en las que se aplica, sea en el ámbito social, económico, político, cultural, empresarial (Caballero, Reyes y Solís, 2006).

En análisis retrospectivo de los principales exponentes del término estrategia se destacan los de Von Neumann y Morgenstern, quienes definen la estrategia empresarial como la serie de actos que ejecuta una empresa, y que a su vez son seleccionados de acuerdo con una situación concreta. Peter Drucker (1954) afirma que la estrategia requiere necesariamente que los gerentes analicen la situación presente en la que se encuentran y que modifiquen lo que sea necesario para mejorar. Su definición parte de la idea de que los gerentes deben saber con qué recursos cuenta la empresa y cuáles debería tener atendiendo a sus propias necesidades. A su vez la primera definición moderna de estrategia empresarial es la contenida en la obra escrita por Alfred Chandler en 1962: Strategy and Structure. Analiza con detenimiento las actividades de cuatro gigantes de la industria estadounidense, de principios del siglo XX, definió la estrategia como el elemento que determina las metas básicas de una empresa, a largo plazo, la adopción de cursos de acción y la asignación de los recursos necesarios para alcanzar estas metas (Mintzberg, Quinn y Voyer, 1997).

La estrategia de una empresa no pasará ninguna de las pruebas si no ofrece una clara orientación a la empresa. Incluso los emprendedores que operan en solitario se pueden beneficiar de una estrategia definida (Bhide, Sahlman, Mc Nell y Block, 2004). 
El estudio de la estrategia ha generado diversas perspectivas y diversas escuelas que se han encargado de analizar el logro y desarrollo de las organizaciones, siempre por medio de la intervención del factor humano, como la escuela prescriptiva, la descriptiva, la de diseño, la emprendedora, la de planificación, la del aprendizaje, del posicionamiento, la cultural, la del entorno y la integradora (Labarca, 2008).

En la década de los setentas Ansoff presenta su matriz en los cuales relaciona los mercados con los productos y los clasifica cada uno como nuevos o existentes, así es posible identificar el cuadrante de penetración en el mercado, desarrollo de productos o diversificación, desarrollo de mercados o diversificación y la diversificación pura. De ahí que pueden identificarse cuatro componentes de la estrategia, ámbito producto - mercado, crecimiento, ventajas competitivas y la sinergia como una aptitud para triunfar en la nueva actividad (Cordero, 2011).

Porter (1980) propone sus cinco fuerzas, marcado las estrategias de liderazgo en costos, concentración de los costos y concentración de la diferenciación.

Wernerfelt (1984) propone su teoría de recursos y capacidades, a través de ella pretende ayudar a la organización a manejar su ambiente y con base en ello incrementar su desempeño, por su parte Barney (1986) y Grant (1991) consideran que esta teoría se encarga de enfatizar la importancia de los recursos internos de la empresa y las capacidades bajo el contexto del ambiente competitivo y dan especial énfasis en el desarrollo de recursos y capacidades organizacionales.

En la última década el término de capital social ha tenido gran auge y ha sido estudiado desde diversas corrientes. Pero hasta hoy en día no existe una definición estandarizada que logre reunir el consenso de todos los investigadores, un primera aproximación lo describe como elementos tangibles que se emplean en la vida diaria de la gente, pudiendo ser la buena voluntad, compañerismo, simpatía y relaciones sociales que se dan entre los individuos, por lo que entre más miembros tenga un grupo social se acumulara más capital social que puede mejorar las condiciones de vida de los miembros de la comunidad (Portela y Neira, 2003).

Roman, Gómez y Smida (2013) ubican el origen del capital social en los trabajos de Hanifan (1916), aunque su significado actual es el resultado de los aportes de Bourdieu (1986). Aunque existen autores que plantean definiciones diferentes, en general se puede afirmar que, entre ellos mismos y los demás eruditos del tema existe consenso alrededor de la idea de que las relaciones sociales son el elemento central de este constructo teórico (Westerlund y Svahn, 2008; Andriessen y Gubbins, 2009).

Para Contreras, López y Molina (2011) el capital social es el que suministra al empresario el apoyo moral lo que a su vez le ofrece modelos que le permiten enfrentar dificultades organizacionales. Se vincula entre el comportamiento de las organizaciones y de la sociedad, fortaleciendo el acceso a diversos recursos tangibles o intangibles.

El capital social consiste en la serie de recursos inherentes a las redes sociales, al igual que el capital humano, el social es considerado como el conjunto de recursos invertidos para la obtención de un beneficio, pero a diferencia del humano que invierte parte de conocimiento y habilidades, el capital social invierte en las redes sociales (GarcíaValdecasas Medina, 2011)

De acuerdo a Ramírez (2005) quien cita a Bourdieu (1986) el capital social es considerado como una herramienta que trata de identificar los efectos sociales, como ejemplo está el caso en que 
dos personas teniendo el mismo capital económico o cultural logran diferentes resultados. Por lo que establece que la diferencia obtenida está vinculada a lo que él denomina «influencias» o «relaciones» traduciéndolas en lo que es la capacidad del individuo para poder interrelacionar a su favor, el capital de un grupo es reconocido como recursos mutuos por lo que para Bourdieu es posible identificar cuando menos cuatro variables que son: 1) el capital social es algo que se puede derivar del individuo cuando este pertenece a un grupo o red social 2) los vínculos que se dan por medio de la existencia de intercambios materiales o simbólicos los que se generan dentro de los citados grupos sociales, 3) la existencia de relaciones interinstitucionales y 4) el conjunto de recursos que el propio grupo posee.

...el capital social, es considerado como factor clave para el fortalecimiento democrático de cualquier país, pues contribuye, entre otros aspectos, al desarrollo institucional, fruto del cual se consigue una mayor y más rápida integración de los países en el desarrollo de un mundo globalizado. Volvemos a comprobar cómo los vínculos entre variables culturales y económicas parecen mayores de lo que el pensamiento neoclásico tomó en consideración. De esta forma las actividades sociales son entendidas como inversiones, en vez de consumidoras de recursos públicos. Como ejemplo se toma el caso de los países nórdicos, en concreto Suecia, heredera de la tradicional socialdemócrata escandinava, donde el Estado financia la creación de asociaciones juveniles, tanto culturales como deportivas, para luego perder su control en beneficio de la sociedad civil, fomentando así la participación política y ciudadana de sus jóvenes, lo que revierte en una mayor integración democrática y cívica de estos sujetos. Es decir, se fortalece el papel del capital social como estructurador de la sociedad, así como elemento imprescindible del análisis económico (Sánchez Jiménez, 2013:491).
De acuerdo a Guia (2000) el capital social se centra en una red de relaciones interpersonales de los individuos lo que al igual que el capital humano se relaciona directamente con la producción de bienes y servicios. El capital social no se encuentra ni en las relaciones ni en los individuos, ni siquiera en los elementos físicos empleados para la producción, sino que es inherente a la estructura social de los individuos, en donde únicamente es en este tejido social que se encuentra realmente el capital social, de esta forma si los miembros de una red social, tienen acceso a una serie de recursos estos dependen de los propios recursos con que cuenta el grupo, es decir del nivel de capital natural, financiero, humano y tecnológico en manos de la red social (Galaso, 2011).

\subsection{Estudios de género}

La figura de la madre es en muchos casos un modelo a seguir, así como la comunicación y la iniciativa son competencias más fuertes de los jóvenes, conclusiones extraídas del informe sobre la cultura emprendedora a edades tempranas (Cartagena, 2014).

Es fundamental ver como las mujeres han cambiado al mundo dentro del ámbito laboral, en términos de emprender y fortalecer el ámbito familiar, dado a que se adentra en el campo laboral y lo han transformado paulatinamente, como se puede constatar en investigaciones empíricas realizadas en varias partes del mundo, mismas arrojan información que permite conocer el crecimiento de la mujer en todos los ámbitos sociales. Dada esta situación se hace necesario enfatizar la importancia que tiene el papel de la mujer en la sociedad, en la economía del siglo XXI las mujeres son una fuerza que ningún país debe ignorar (Bernal, 2013).

Grabinsky y Zabludovsky (2001) destaca que no se requiere ser futurologo para poder observar 
el cambio radical en la situación de la mujeres en el siglo XXI, ya que en el siglo XX se obtuvieron grandes beneficios como el derecho al voto, a ser electas, trabajar en centros de educación, entre otros muchos roles sociales, con base en estos antecedentes se puede proyectar que el papel de la mujer en sera fundamental con el paso del tiempo.

De acuerdo con datos del Instituto Nacional del Emprendedor, al 2013 el 19 \% de los emprendedores del país eran mujeres, lo cual se traduce en cerca de 4 millones de emprendedoras. Por lo que el crecimiento del emprendimiento femenino ha presentado una evolución natural en razon del papel de la mujer como parte de la fuerza laboral. En México por definición son emprendedoras, y lo hacen como forma de incremetar el ingreso familiar (Rodríguez, 2013).

En los últimos quince años se ha observado la entrada de muchas mujeres de todas las edades y condiciones sociales, casadas, viudas, divorciadas o solteras al frente de pequeñas empresas. Y aunque se ha difundido mucho de que la crisis de los ochenta fue la que orilló a muchas mujeres a buscar ingresos y trabajar, tambien estamos presentaciando un cambio fundamental de actitud que, es ya irreversible (Grabinsky y Zabludovsky, 2001: 27-28).

En nuestros días la particiación de la mujer en el ámbito laboral va aumentando paulatinamente, en el caso de México en las últimas cuatro decadas han aumentado su participación en el sector económico, para el 2009 las mujeres trabajadoras representaban un tercio de la población económicamente activa del país, y como empleadoras la quinta parte en todo el país lo que hace suponer que nos enfrentamos a un crecimiento de mujeres emprendedoras (Zárate, Vega y Zárate, 2013).

El notable incremento de mujeres que trabajan fuera del hogar, es considerado como una de las tranformaciones sociales de mayor significancia hoy en día. En México de tener en 1970 un 21 \% paso al $38 \%$ en el 2010, identificandose un aumento en las zonas urbanas, en especifico en el sector servicio, en donde el numero rebaza consierablemente al de los hombres (Zabludovsky, 2013).

Los antecedentes de la actividad empresarial han mostrado varias circunstancias que surgen en torno a la realidad de la incorporación de las mujeres al sector empresarial. Aparecen ciertos conflictos cuando comienza a considerarse que la mujer está desempeñando una actividad tradicionalmente masculina, esto es, dominada por los hombres, Aunado a esto, afloran algunos cuestinamientos sobre el desempeño de las mujeres y la multifuncionaliad tradicional que deberá cubrir, como empresaria, esposa y mujer (Molina, Contreras, y López, 2014: 278).

Según Flores-Estrada (2007) en el 90 \% de los países, es mayor el número de mujeres en el sector de servicios. Por otra parte en todos los países, con excepción de Honduras, Macao, China, las Islas Maldivas y Marruecos, el número de hombres empleados en la indistria es superior a un que el de las mujeres.

Durante las últimas tres decadas, la participación de las mujeres en el mercado laboral y empresarial se ha incrementado de manera notable, no obstante, la atención de los especialistas se ha centrado en la participación de lass mujeres asalariadas y no en las que se dedican a las actividadees empresariales, por lo cual en el terreno de las ciencias sociales de México y América Latina existe una insuficiete información sobre el tema. Esta limitación ha sido señalada tanto en los trabajos que estudian a las mujeres empreadoras (De la Paz,2009); Villagómez, 2004; Serna, 2001: 91-92; Zabludovsky, 1993b, 1998, 2001 a) como en los que se encuentran en las microempresas en los nivles de subsistencia (Suarez y Bonfil, 2004:12) (Zabludovky, 2013:43)

La organización internacional del trabajo (OIT, 1998) afirma que las mujeres tienen aproxima- 
damente una decada en que se encuentran mejor preparadas y que ocupan mejores puestos de trabajo, esto se puede apreciar desde el momento de la formación académica, ya que cada dia son más las mujeres que se encuentan en el aula desde el momento de su formación profesional y este fenomeno puede aprecierse en todo el mundo (Zárate, Vega y Zárate, 2013).

En cuanto al estudio de las mujeres emprendedoras, de acuerdo a Gutiérrez (2012) las grandes compañías reconocen la aportación de las ejecutivas de alta dirección en sus empresas y equipos de trabajo, debido a que han demostrado buenos resultados.

Analizando el trabajo realizado por mujeres en las últimas décadas en 17 países europeos y México, se observa que existe un crecimiento en la tasa de participación laboral. Un aspecto por destacar de este crecimiento es que se ve reflejado en situaciones económicas desfavorables en el aspecto económico, lo que no permite la absorción de mano de obra disponible. A su vez, el Banco Mundial (BM) considera que uno de los factores más importantes es el feminismo, ya que no genera sus planteamientos en razón de todas las mujeres y todos los países, toma en cuenta las costumbres, las instituciones sociales, las políticas y el desarrollo económico, que permitan establecer medidas para atacar la disparidad de género. Por lo que propone una determinada estrategia que reta los ámbitos descritos anteriormente concentrándose en los siguientes apartados: La falta de información genera discriminación en al ámbito laboral, que va desde el nivel macro y micro, en razón del primer nivel la ofertas de empleo dirigidos a la mujer y en el segundo sobre la productividad de la misma, por lo que recae en el estado la obligación de ampliar la información en cuanto a capacitación y experiencia de ellas, a fin de promover el trabajo femenino.
- Se deberá considerar el potencial del sector.

- La desigualdad de género se da en mayor medida entre la clase baja, dentro de los diversos paises (Chávez, Chapoy, Rueda, Gonzáles y Rodríguez, 2010).

Tambunan (2009) señala que en Asia el tema del desarrollo del espíritu empresarial de la mujer tiene limitaciones, para poder mantenerse o crecer no obstante que las PyMEs son de gran importancia es este país ya que representan el $95 \%$ de todas las empresas, lo que puede proporcionar una mejor calidad de vida a más del $90 \%$ de la fuerza laboral del país. Por otro lado, las mujeres emprendedoras se encuentran principalmente generando microempresas que son consideradas como de actividades tradicionales y de baja generación de ingresos.

Espinosa, De León y Zarur (2015) afirman que el 54 \% de las empresas están encabezadas por mujeres en México y se encuentran dentro del sector servicios, el $31 \%$ dedicadas al comercio y solo el $15 \%$ estan ubicadas en la industria y el sector agropecuario.

Revisando la información proporcionada por la Asociación Mexicana de Mujeres Empresarias (AMMJE) se obtienen los siguientes datos:

En uno de cada cuatro hogares mexicanos tiene como jefa a una mujer y el 70,5\% cuenta con un aporte de ingresos femeninos, según datos del Censo de Población y Vivienda 2010. Estas cifras ayudan a entender la motivación que tienen algunas mujeres para emprender, ya sea por la falta de empleo, necesidad de complementar el gasto familiar e incluso, para tener horarios que les permitan atender a sus familias (Maubert, 2013, p.1).

Existe una diferencia en cuanto al número de mujeres emprendedoras y Maubert menciona lo siguiente: 
La mexicana actual ya no sólo se encarga del hogar o su familia. Hoy representa más de una tercera parte de la población económicamente activa del país, y las que trabajan por su cuenta o tienen un negocio son cada vez más. Con un estilo de liderazgo flexible, cooperativo Y conciliador, las emprendedoras y ejecutivas enfrentan todavía muchos retos... pero están transformando sus empresas, sus mercados y nuestra manera de pensar (Maubert, 2013, p.1).

En investigaciones empíricas como las realizadas por Maubert (2013), Gómez, Delgado y Vidal (2010), entre otros, se ha estudiado a las mujeres mexicanas emprendedoras, de igual forma esto se puede constatar en el entorno social en el que se encuentran, dado que muy seguramente la «tiendita» que se encuentra en la colonia está dirigida por una de ellas, la estética a la cual se acude a cortarse el cabello es atendida y administrada por una mujer, solo por mencionar algunos de los negocios en los que emprenden.

Gutiérrez (2012) afirma que las mujeres cada vez más están ocupando puestos de mayor jerarquía y relevancia, la mujer está comenzando a desempeñar roles de impacto como son los puestos de operaciones y ventas. América Latina se considera como una de las regiones con un mayor índice relacionada a la actividad emprendedora, llegando a 21 \% en relación al 12,2 \% de países de Asia y Europa considerados con un ingreso bajo o medio y el 7,9 \% de los considerados como de alto nivel de ingresos. Por lo que las empresas de mujeres en América Latina se consideran como contribuyentes dinámicos de la economía del país en donde generan sus empresas. Estudios llevados a cabo por la Fundación Nacional para Mujeres Propietarias de Empresas determinaron que en América Latina puede llegar hasta $19 \%$ su contribución en el Producto Interno Bruto (PIB), no obstante, las empresas formadas por mujeres pueden llegar a tener una serie de limitantes. Esto debido a que América Latina posee un alto índice relacionado a los fracasos de negocios dirigidos por mujeres (Powers y Magnoni, 2010).

Derivado de la información anterior es que se hace necesario llevar cabo la presente investigación, la cual tiene como principal objetivo el poder describir la trayectoria y estrategias empleadas por mujeres emprendedoras, si bien es un tema que se has estudiado desde diversas perspectivas, como lo muestran investigaciones realizadas en España por Fuente y Sánchez (2010) analizan el perfil emprendedor desde una perspectiva de género, igualmente en España estudios de Ortiz, Duque y Camargo (2008) han desarrollado investigaciones similares. En Argentina, Caracciolo y Foti (2013), han realizado estudios de la mujer en la economía social y solidaria. En México se ha estudiado a la mujer y el emprendimiento, así como la influencia de la edad, el género y la escolaridad en el emprendimiento realizados por Escamilla, Caldera y Ortega (2010), de aquí que surge la iniciativa de estudiar la trayectoria y estrategias implementadas por mujeres emprendedoras.

Por lo que a la revisión de la literatura se refiere se puede apreciar que en diversas partes del mundo la mujer ha pasado de ser el eje de la familia, a formar parte del eje de la economía. Lamentablemente, el papel que juega en el ámbito laboral aun en pleno siglo XXI no es visto con la igualdad y relevancia que amerita, ya que se puede apreciar la discriminación que existe para el trabajo de la mujer, lo que en la mayoría de las ocasiones propicia que sea ella mismas la creadora de su propio medio de subsistencia, para poder así contribuir al sostenimiento de la familia, y con ello fortalecer la economía de las naciones.

\section{Método}

El método utilizado fue con un enfoque cualitativo, a través del paradigma interpretativo 
de la teoría fundamentada, con el fin de crear teoría que explique el fenómeno de estudio (Vivar, Arantzamendi, López - Dicastillo y Gordo, 2010).

Fueron entrevistadas 40 mujeres emprendedoras de las cuales 34 eran fundadoras de la empresa y 6 fungen como encargadas de las mismas. La edad de la mismas tuvo una media de 52,75 años con una desviación estándar de 10,31, y la trayectoria empresarial fue de 19,3 años con una desviación estándar de 12,06; de igual forma afirmaron 25 de ellas estar casadas, 2 viudas y 13 no quisieron declarar su estado civil, asi mismo 22 declararon ser profesionistas, 2 con carreras técnicas una con educación básica y 13 no quisieron decir su nivel de estudios, el giro en el que se mueven las empresas fueron 12 del giro comercial, 6 de manufactura y 22 de servicios.

El proceso de análisis se realizó en dos niveles, el textual que tuvo como objetivo tener una familiaridad con los contextos establecidos, identificar las posibles unidades de análisis considerando los temas abordados, localizar las categorías emergentes y determinar la frecuencia de la presencia de los códigos. El segundo nivel fue el análisis conceptual, el cual se realizó con la finalidad de acotar los códigos abiertos a través de la generación de familias, relacionar los datos de forma que se lograra una comprensión de los datos, lo que lleva a la fundamentación de la investigación y realizar una propuesta de teoría a fin de poder identificar lo que sucede con las mujeres emprendedoras.

Se localizaron 43 códigos de los cuales 5 de ellos se consideraron primarios debido a su frecuencia de aparición, estos fueron, el análisis de los competidores (58 citas), experiencia en el ramo (50 citas), recursos con los que se contaba (49 citas), estrategias para entrar en el mercado (48 citas) y motivo (48 citas), las familias de códigos encontradas fueron barreras (82 citas), capacidades (194 citas), capital comercial (262 citas), capital cultural (259 citas), capital financiero (113 citas), capital simbólico (226 citas), capital social (266 citas), capital tecnológico (23 citas), clientes (183 citas), competidores (106 citas), productos sustitutos (35 citas), proveedores (66 citas), recursos (161 citas).

Con estas familias se conformaron 3 familias generales, las que obedecen a la teoría de las cinco fuerzas de Porter, clientes, proveedores, el mercado, competidores, barreras de entrada y productos sustitutos, las que obedecen a la teoría de recursos y capacidades de Wernerfelt, recursos, capacidades y experiencia y finalmente las que obedecen a la teoría de los capitales organizacionales, el comercial, cultural, financiero, simbólico, social y tecnológico.

\section{Resultados}

A continuación, se presenta el análisis conceptual generado con ayuda del software Atlas Ti, con base en cada una de las tres teorías analizadas, para las cinco fuerzas de Porter, el análisis queda: (Figura 1).

Es claro identificar que para este caso la teoría de Porter se confirma ya que se muestra clara relación entre las cinco fuerzas, formando un centro fuerte en lo que respecta al código clientes.

En cuanto a la teoría de los recursos y las capacidades, la relación quedo: (Figura 2).

Nuevamente es fácil identificar que la teoría de recursos y capacidades de Wernerfelt de confirma la estar los dos elementos perfectamente definidos y solamente asociados en sus bases teoricas, es decir, en los recursos y las capacidades.

Finalmente para la teoría de los capitales organizacionales, la relación entre familias quedo: 


\section{Figura 1. Red de familia de códigos de las cinco fuerzas de Porter}

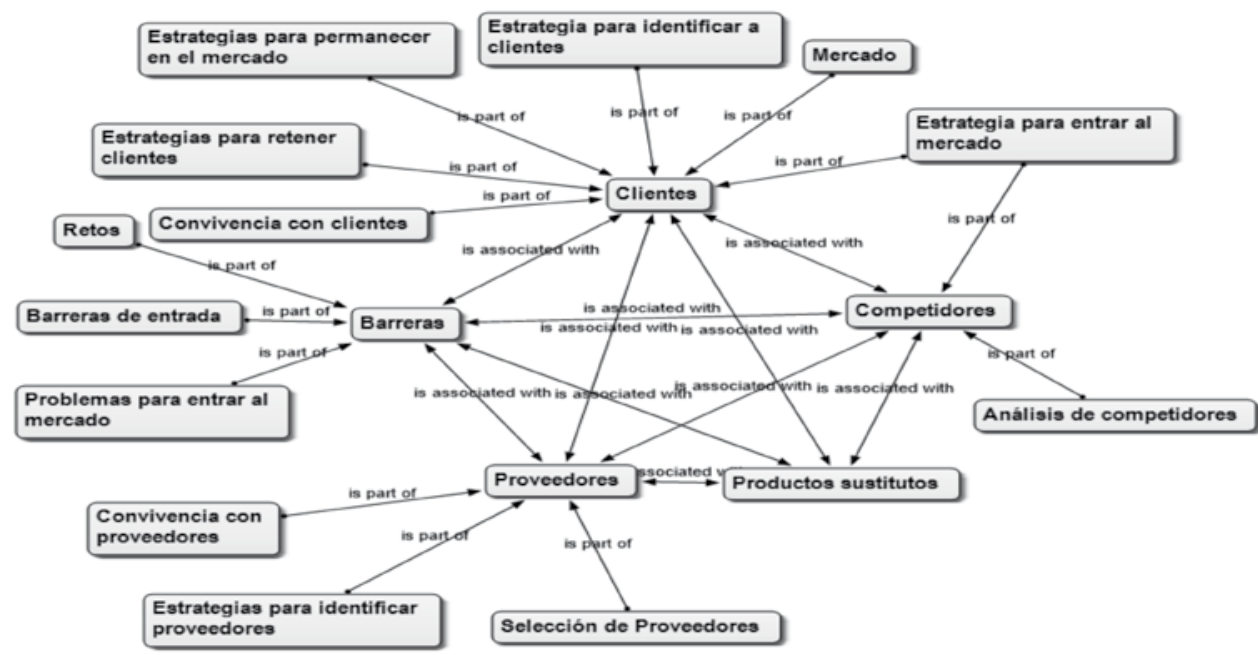

Figura 2. Relación de los elementos de familiad de codigos de recursos y capacidades

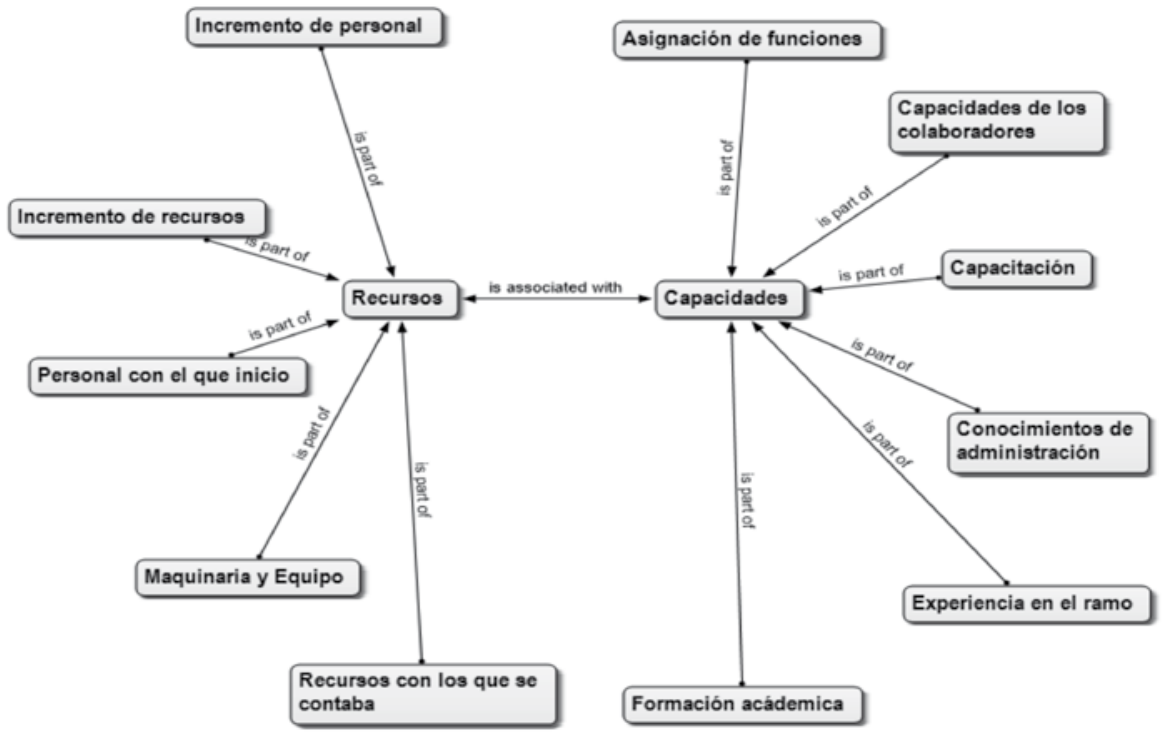




\section{Figura 3. Relación entre los elementos de familia de códigos de capital organizacional}

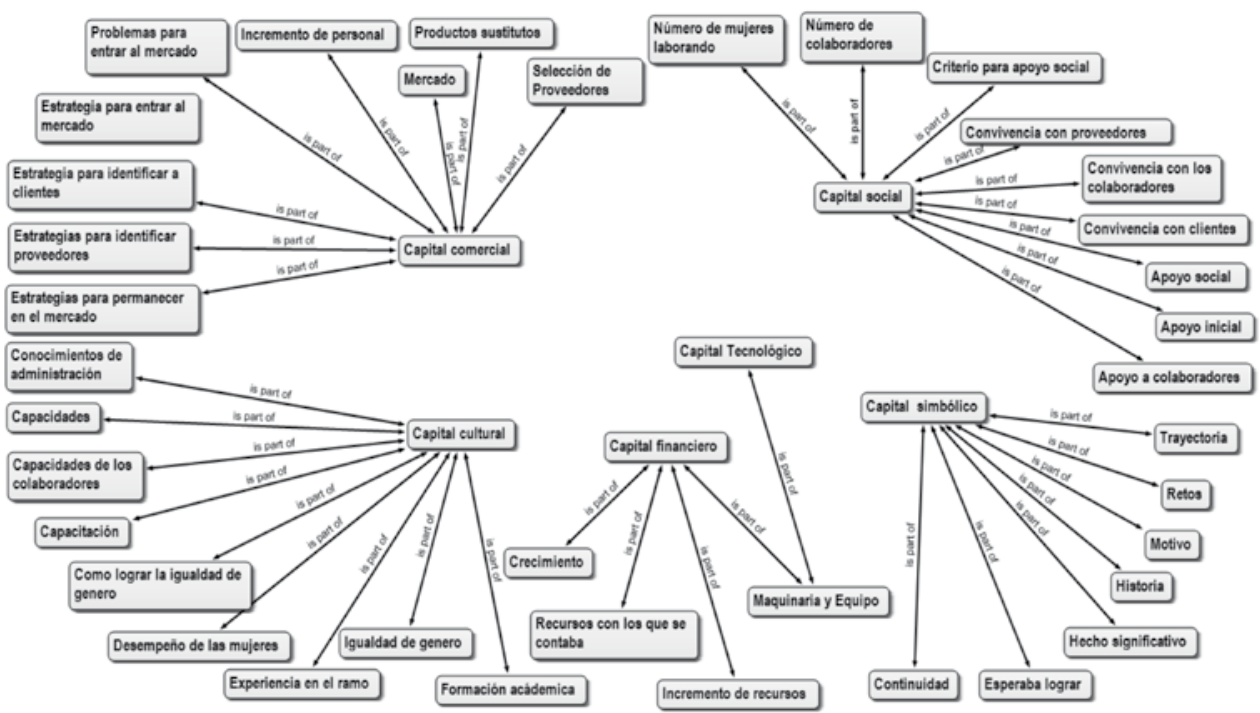

Fuente: Elaboración propia.

En este caso es de llamar la atención que los capitales organizacionales estan desvinculados unos de otros, a excepción del capital financiero y el capital tecnológico que se vinculan a través del código de maquinaria y equipo, fuera de este, el resto de códigos aparecen aislados unos de otros sin que al parecer tuvieran alguna relación, como lo plantea la teoría.

\section{Conclusiones}

En el transcurso de la investigación fue posible confirmar lo establecido por Stevenson (2004) en el sentido de que el emprendedor busca busca e identifica oportunidades, ya que la entrevistadas evidenciaron su capacidad e impulso para desarrollar sus empresas y contribuyeron a mejorar y hacer más eficiente el mercado por medio de la eliminación de turbulencias y la generación o creación de nuevas riquezas, como lo establecía Cantillon (1755).
Las entrevistadas demostraron poseer, operar y tomar riesgos, al mismo tiempo que procesan y ejecutan empresas con fondos de diferentes fuentes, y que identificaron necesidades insatisfechas del mercado para poder ofrecer un producto o servicio que cubriera dichas necesidades (Greene, 2011).

Las emprendedoras entrevistas fueron capaces de introducir ideas, productos y servicios dentro del mercado, desarrollando estrategias que rompieron con los esquemas tradicionales, combinando eficientemente conceptos de bajo coste y factores diferenciales.

Al respecto se puede describir la existencia de cinco tipos de estrategias implementadas por las mujeres emprendedoras. La primera es una estrategia de penetración en el mercado, 12 de las 40 entrevistadas realizaron un análisis con el fin de precisar su mercado y poder penetrar de la mejor manera, las estrategias utilizadas no responden a la implementación de alguna teoría 
en particular, sin embargo, se puede evidenciar el uso de la llamada «intuición» en la puesta en práctica de estas estrategias.

El segundo grupo de estrategias utilizadas fue para identificar a los clientes, se observa la preocupación por identificar las características de los mismos, el uso de estudios de mercado, o el uso de la experiencia en el ramo, sin embargo, en 15 de los 40 casos no existe una estrategia definida, sino que van adecuando el producto o servicio al cliente que se los requiere, situación que demuestra que pese a no contar con una estrategia, vuelve a prevalecer la intuición.

El tercer grupo fueron las estrategias para identificar proveedores, dentro de las cuales destacaron las características de calidad, precio y condiciones de entrega; aun así, el factor de intuición vuelve a hacerse presente ya que al menos dos emprendedoras no declararon tener un proveedor definido

Otro grupo de estrategias estuvo identificado como las que se emplean para permanecer en el mercado, sobresalieron las estrategias de ofrecer productos y servicios de calidad, mejorar el precio de la competencia, ofrecer promociones y estar al pendiente de los clientes; en cuanto a esta última puede precisarse que las emprendedoras se adaptan a las necesidades del cliente, le ofrecen un saco a la medida para lograr la plena satisfacción del cliente y poder permanecer en el mercado. Algunas de estas estrategias son implementadas de forma inconsciente, es decir, nuevamente, por la sola intuición.

El último grupo identificado fueron las estrategias para retener a los clientes, el $90 \%$ de las entrevistadas dijeron emplear algún tipo de estrategia para retener a los clientes, talas como el ofrecer un buen trabajo, el precio, el servicio, la calidad, el buen trato y la variedad de productos o servicios que ofrecen.
Con base en lo anterior es posible afirmar que las decisiones sobre las estrategias se fundamentan más en el criterio y el análisis de los objetivos, que en la experiencia o la intuición de los que dirigen la empresa (Drucker, 1954; Mintzberg, Quienn y Voyer, 1997), sin embargo es posible llegar a situaciones en las cuales los emprendedores consideran la escala del posicionamiento, y entienden que la estrategia es más perspectiva que posición, lo que permite proteger las capacidades de la organización, por lo que la estrategia resultante si bien es deliberada, no siempre es consciente (Labarca, 2008).

La presente investigación permitió conjuntar las tres teorías presentadas e identificar, con base en ellas, descriptores que puedan explicar los factores relacionados con el emprendimiento en las mujeres (Figura 4).

Las entrevistadas conceptualizaron cada uno de los factores que comprenden el modelo, para lo cual se considerara un resumen de cada uno de ellos que en apartado de análisis se desarrolló a detalle.

Capacidades: las consideran el conjunto de aptitudes que tiene las emprendedoras para salir adelante. Consideran otras constantes como el entusiasmo, la disposición al trabajo, el hacer lo que le gusta, vinculándola a la formación académica que pueda llegar a tener la emprendedora.

Capacidades de los colaboradores: consideran importante que las personas tengan conocimientos previos, tampoco es un factor determinante para tenerlos como colaboradores. Una de las cualidades que destaca es que buscan personas que tengan disponibilidad al trabajo, para ellas no es necesario que los colaboradores cuentas con capacidades bien definidas al momento de laborar en sus empresas, ya que ellas están en la disposición de apoyarlos mediante la capacitación. 


\section{Figura 4. Factores relacionados con el emprendimiento en mujeres}

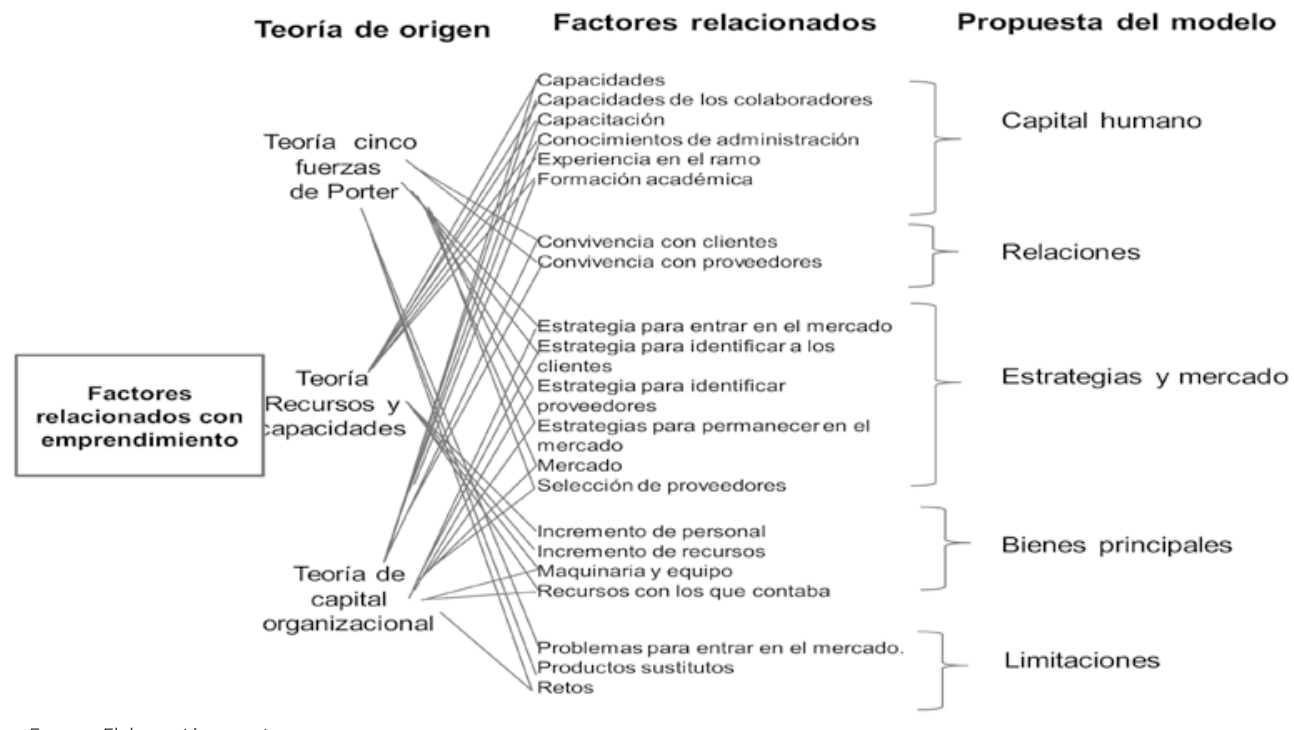

Fuente: Elaboración propia.

Capacitación: Considera tres vertientes, primero la capacitación que se brinda a sus colaboradores a fin de que puedan desarrollar mejor su trabajo, segundo, la capacitación que las mismas emprendedoras han recibido a fin de poder brindar un mejor servicio a sus clientes, finalmente la capacitación directa en el área de trabajo.

Conocimientos de administración: se hace referencia a que pueden no tener una formación profesional en el área, pero que con base en la experiencia adquieren la habilidad para ejecutarla de la mejor manera, o bien apoyándose de expertos que les faciliten si ejecución.

Experiencia en el ramo: no es un factor determinante el contar con experiencia en el ramo, si contribuye al desarrollo de su empresa, pudiendo también optar por relacionarse con expertos.

Formación académica: no es necesario contar con una formación académica, pero no descartan que esta les ayuda a desempeñar mejor su función.
Convivencia con clientes: es de suma importancia para las emprendedoras, reconocen que de los clientes depende el éxito de su empresa, por lo que prestan especial atención.

Convivencia con proveedores: destaca que la relación con los proveedores debe ser de respeto y cordialidad, sin descuidar lo que el proveedor debe de cumplir con sus exigencias en cuanto a calidad y plazo de entrega.

Estrategias para entrar al mercado: en este apartado se encuentran las que actúan con base en la intuición, otra estrategia es satisfacer las necesidades de un grupo reducido de clientes, emplean los vínculos con familiares y amigos a fin de poder dar a conocer sus productos o servicios, finalmente consideran importante diseñar una estrategia específica atendiendo a las características del producto o servicio.

Estrategias para identificar clientes: sobresale la necesidad de establecer estrategias diseñadas 
atendiendo las características de la empresa y las necesidades del cliente, sin dejar de lado el empleo de la intuición.

Estrategias para identificar proveedores: la estrategia que mayormente se emplea es la de seleccionar a los proveedores atendiendo a la calidad, el precio, las condiciones de vente entre otras.

Estrategia para permanecer en el mercado: ofrecer productos de calidad, competir con el precio, el servicio y los requerimientos del cliente.

Selección de proveedores: no existe un mecanismo definido para seleccionar a sus proveedores, se puede visualizar que existe una relación constante por considerar como factor determinante la calidad, el precio, las condiciones de entrega principalmente.

Mercado: destaca la importancia de conocer su mercado, sea desde antes de iniciar operaciones o bien identificarlo lo más pronto posible empleando la intuición.

Incremento de personal: se hace referencia que a lo largo de la vida de la empresa han logrado mantener el número de colaboradores o bien han podido incrementarlo, lo que a su vez contribuye a la generación de fuentes de empleo, el desarrollo de la economía local, regional e incluso nacional.

Incremento de recursos: se consideran el incremento de todo tipo de recursos, desde el humano, material, económico, etc.

Maquinaria y equipo: se entiende como el incremento de recursos materiales, que se da a través de los años, este factor se relaciona de forma directa con el incremento de recursos, que genera mejores condiciones laborales.
Recursos con los que contaba: se podemos englobar todos los recursos con los que se inició, ya sean materiales, humanos, financieros.

Problemas para entrar en el mercado: son las problemáticas que van desde no poder llegar a acuerdos con los clientes, el cumplir con la realización de trámites, la falta de capital financiero, destacando la problemática para entrar al mercado derivado del género, es decir han presentado problemas para entrar al mercado por el simple hecho de ser mujeres, en un mercado ocupado en su mayoría por hombres, los problemas para compartir tiempo familia - empresa - familia, y la problemática generada por la misma competencia.

Productos sustitos: las emprendedoras demuestran que conocen su mercado, identifican aquellos productos que pueden llegar a sustituir lo que ellas ofrecen. La mayoría de las entrevistadas conocen aquellos productos o servicios que pueden ser sustitutos, ya sea por la cercanía de los competidores o bien porque hacen análisis constantes para poder estar al tanto de las condiciones del mercado.

Retos: comprenden las dificultades que surgieron al inicio de su empresa, que van desde no contar con clientes, ganarse la confianza del cliente, conocer el mercado, lidiar con la competencia, contar con la capacidad instalada, pero el que destaca el lograr la preferencia del cliente por brindar un producto o servicio de calidad.

El modelo descrito refleja la información que permitió identificar los factores relacionados con el emprendimiento en mujeres, sin perder de vista que pueden ser relativos, debido a que se consideran las características del grupo en el cual se obtuvo la información. 


\section{Bibliografía}

Andriessen, D. \& Gubbins, C. (2009). Metaphor analysis as an approach for exploring theorical concepts: The case of social capital. Organization Studies, DOI: https://doi.org/10. $1177 / 0170840609334952$

Aurieu, A. (2003). WindRed.com ideas y negocios en red. Recuperado el 29 de 09 de 2013, de http://148.206.107.15/ biblioteca_digital/articulos/9-223-2822qqp.pdf

Barney, J. (1986). Stretegic factor markets: expectations, luck, and the theory of business stretagy, Management Science, 32, $1512-1514$

Baro, M. (2012). Percepciones sobre capital social en Argentina. Global Media Jounal, 9(17), 1-17.

Bernal, G. M. (2013). Empresarias Ejecutivas en México Diagnósticos y desafíos. México: IPADE.

Betancourt, G. B. (2004). La Capacidad emprendedora y el desarrollo de nuevas empresas. Cuaderno de Administración, 20(32), 107-136.

Bhide, A., Sahlman, W. A., Mc Nell, S. J., \& Block, Z. (2004). Ideas con impacto Harvard Business Review La iniciativa emprendedora. (M. E. Aparicio, Trad.) Ehttps: //engage. intel.com/servlet/JiveServlet/previewBody/33618-102-1-40804/Leccion_1_Guia_de_Aprendizaje. pdf, Barcelona, España: Deusto.

Bourdieu, P. (1986). The forms of capital. En Richardson, J. G. (Ed.), Handbook of heory and research for the sociology of education. New York: Greenwood.

Bruinet, I., \& Alarcón, A. (2004). Teoría sobre la figura del emprendedor. Revista de Sociología (73), 81-103.

Bustos, S. W. (2005). Cuestiones del conocimiento: Hayek, Keines y más alla. (ESEADE, Ed.) Revista Libertas XII: 43, XII (43), 1. Obtenido de http://www.eseade.edu.ar/files/Libertas/3_7_Butos.pdf\#page=15\&zoom=auto,-107,823

Caballero, A., Reyes, R., \& Solis, P. P. (2006). La estrategia competitiva que adopta la empresa transnacional en el mercado global. Redalyc.org (220), 89-121.

Cantillon, R. (1755). Essai sur la nature du commerce en general. Macmillan, London, UK.

Caracciolo, M. \& Foti, M. (2013). Economía social y solidaria, aportes para una visión alternativa. Recuperado de: http://base.socioeco.org/docs/ess-una-vision-alternativa.pdf
Cartagena, A. d. (2014). Actualidad municipal. Recuperado el 22 de 04 de 2014, de Toda la información sobre el ayuntamiento de Cartagena: http://www.cartagena.es/frontend/ genericas/detalle_noticia/_Mq4DsbcKUSLlk8jhdoxXts_ ZOXJq5XaHzyC80hoC7q6300ve8CxXlg

Casco, C. J., \& Barrera, M. F. (2011). Como crear 1000000 de nuevos emprendedores en tu país. España: emprendedorex. com

Chandler, A. (1962) Strategy and Structure: Chapters in the History of American Enterprise. MIT Press, Boston.

Chávez, H. M., Chapoy, B. A., Rueda, P. I., González, M. M., \& Rodríguez, L. P. (2010). Trabajo Femenino, La nueva desigualdad. México: Universidad Nacional Autónoma de México, Instituto de investigaciones Económicas.

Contreras, S. R., López, S. A., \& Molina, S. R. (2011). Emprendimiento dimensiones sociales y culturales en las Mipymes. México: Pearson.

Cordero, A. (2011). Scielo Perú. Recuperado el 10 de 04 de 2014, de http://www.scielo.org.pe/scielo.php?script=sci_art text\&pid=\$2077-18862011000100006

Crissien, J. (2009). Investigando el entrepreneurship tras un marco teórico y su aporte al desarrollo económico de Colombia. Recuperado de http://www.google.com.mx/url?sa=t\&rct=j $\& \mathrm{q}=\&$ esrc $=s \&$ source $=$ web $\& \mathrm{~cd}=1$ \&ved=0CCsQFjAA\&url =http $\% 3 \mathrm{~A} \% 2 \mathrm{~F} \% 2$ Fjournal.ean.edu.co $\% 2$ Findex.php $\% 2 \mathrm{~F}$ Revista\%2Farticle\%2Fdownload\%2F473\%2F460\&ei=1_ V6Uub3Ns-tqQGUqoH4Cw\&usg=AFQjCNF-7IHtjZX2npon_MtBxYZqX18vTw\&bvm=bv.55980276,d.aWM

Ekelund, R., \& Henert, R. (2014). A history of ecomic theory and methed, (6a ed.). Waveland Press.

El Economista América (2013). El 19\% de los emprendedores en México son mujeres. Recuperado de http://www.eleconomistaamerica.mx/sociedad-eAm-mexico/noticias/5003833/07/13/El-19-de-los-emprendedores-enMexico-son-mujeres.html.

Enciso-Congote, J. D. (2010). El emprendimiento y el bien común: ¿competencias complementarias o excluyentes?. Educación y Educadores, 13(1), 63-76. Obtenido de http://www. redalyc.org/articulo.oa?id=83416264005

Escamilla, Z., Caldera, D. \& Ortega, M. (2012). Por qué las mujeres potencialmente emprendedoras se convierten en empresarias. XVII Congreso Internacional de Contaduría, Administración e Informática. 3 - 5 Octubre 2012. http:// congreso.investiga.fca.unam.mx/docs/xvii/docs/C31.pdf 
Espinosa, I. E., De León, J. S., \& Zarur, O. A. (2015). Género y empresa familiar. El ron no reconocido de la mujer en la directriz de la empresa familiar. Una historia de vida. En S. Z. Cruz, C. O. Lozano, M. G. Ramírez, \& Z. S. Yulieth, MiPyMEs, empresas familiares y organizaciones emergentes, experiencias en México, Guatemala y España (págs. 159188). Chiapas: Universidad Autónoma de Chiapas.

Filion, L. J. (2003). Emprendedores y propietarios dirigentes de pequeña y mediana empresa (PME) (3era ed.). (C. L. López, Trad.) Quebec, Canadá.

Flores Estrada, M. (2007). Economía del género. El valor simbólico y económico de las mujeres. Editorial UCR. http://biblioteca.clacso.edu.ar/Costa_Rica/iisucr/20120725012806/economia.pdf

Formichella, M. M. (2004). El concepto de emprendimiento y su relación con la educación, el empleo y el desarrollo local. Buenos Aires: Instituto Nacional de Tecnología Agropecuaria.

Fuentes, F. \& Sánchez, S. (2010). Análisis del perfil emprendedor: una perspectiva de género. Estudios de Economía Aplicada. Vol. 8, No3, pp. 1 - 28

Galaso, R. P. (2011). Capital social y desarrollo económico Un estudio de las redes de innovación en España. Madrid, España: Universidad Autónoma de Madrid. Obtenido de https://repositorio.uam.es/bitstream/handle/10486/6639/39410_ Galaso_reca_pablo.pdf?sequence $=1$

García- Valdecasas Medina, J. I. (2011). Una definición estructural de capital social. Revista Hispana para el Análisis de Redes Sociales (págs. 1320-160). Redes.

García, G. L. (2008). Condicionantes del emprendimiento en Colombia. El cuaderno Escuela Ciencias Estratégicas, 2(4), 167-185. Obtenido de http://www.google.com.mx/url?sa=t $\& \mathrm{rct}=\mathrm{j} \& \mathrm{q}=\& \mathrm{esrc}=\mathrm{s} \&$ source $=$ web $\& \mathrm{~cd}=1 \& \mathrm{ved}=0 \mathrm{CBwQFjA}$ A\&url=http\%3A\%2F\%2Fdialnet.unirioja.es\%2Fdescarga \%2Farticulo\%2F2991245.pdf\&ei=C36pVPeZKoWGyQTs woGgAw\&usg=AFQjCNGceb-t9oMNBzoSltUlkkyXaCgsp Q\&bvm=bv.82001339,d.aWw

García, P. d., Moreno, C. I., \& Rosique, B. M. (2012). Aptitud emprendedora de los alumnos de Educación Secundaria Obligatoria y Bachillerato en la Región de Murcia. Cartagena. Obtenido de http://www.upct.es/contenido/destacados/ficheros/ 2176Aptitudes-emprendedoras.pdf

Gartner, W. (1988). Who is an entrepreneur? Is the wrong question. Entrepreneurship, Theory and Practice. DOI: https:// doi.org/10.1177/104225878801200401
Gómez, S. Delgado, J \& Vidal, M. (2010). Propuesta de aplicación de la teoría sociológica institucional como marco teórico para el análisis del emprendimiento corporativo. Recuperado de https://www.google.com.mx/search?q=investigaciones+s obre+emprendimiento+en + mujeres $\& i e=u t f-8 \& o e=u t f-$ $8 \&$ rls=org. mozilla:es $-M X$ : official $\&$ client=firefoxa\&channel=np\&source=hp\&gws_rd=cr\&ei=TgRzUpukKe qssASq5YCgBA\#channel=np\&psj=l \&q=teor\%C3\%ADa+d e+emprendimiento++de+R.+Cantillon\&rls=org.mozilla:esMX:official\&start $=20$

Grabinsky, S., \& Zabludovsky, G. (2001). Mujeres, empresa y familia. México: Del verbo emprender.

Grant, R. (1991). The resource-based theory of competitive advantage: implications for strategy formulation. California Management Review 33 (3) pp.114-135.

Greene, C. (2011). Entrepreneurship Ideas in action (5ta ed.). USA: CENGAGE, Leanig

Guia, J. J. (2000). Implicaciones del Capital Social para la Ventaja Competitiva de la Empresa en un Contexto Evolutivo. Catellón de la Plata, España: Universitat Jaume I. Obtenido de http:// revista-redes.rediris.es/webredes/textos/capital_evolutivo.pdf Gutiérrez, M. G. (2012). Guerra por las mujeres. Expansión (1097), 83.

Hanifan, L. J. (1916) The rural school community center Annals of the American Academy of Political and Social Science 67:130-138.

INEGI (2013) boletín de prensa núm. 321/13, resultados de la encuesta nacional de ocupación y empleo cifras durante el segundo trimestre de 2013. Recuperado de HTTP:// WWW.INEGI.ORG.MX/INEGI/CONTENIDOS/ESPANOL/ PRENSA/COMUNICADOS/ESTRUCBOL.PDF

INEGI (2019). Censos Económicos 2019. Recuperado de: inegi.org.mx/programas/ce/2019/

Jaramillo, L. (2008). Emprendimiento: Concepto básico en competencias. Recuperado de http://guayacan.uninorte.edu. co/divisiones/iese/lumen/ediciones/7/articulos/emprendimiento.pdf

Labarca, N. (2008). Evolución del pensamiento estrategico en la formación de la estrategia empresarial. Scielo, 24 (55).

Leiva, B. J. (2007). Los emprendedores y la creación de empresas. Costa Rica: Editorial Tecnológica de CostaRica.

Marulanda, M. J., Correa, C. e., \& Mejía, M. L. (2009). Emprendimiento: Visiones desde las teorías del comportamiento humano. Marulanda Montoya, Jorge Andrés; Correa Calle, 
Geovanny; Mejía, Luis Fernando (66), 153-168. Obtenido de http://www.redalyc.org/articulo.oa?id=20620269008

Maubert, I (2013). Cómo son las emprendedoras mexicanas. Recuperado de http://www.soyentrepreneur.com/21918como-son-las-emprendedoras-mexicanas.html

Mintzberg, H., Quinn, J. B., \& Voyer, J. (1997). El Proceso estratégico conceptos, contextos y casos (1era ed.). Pearson Prentice Hall.

Molina, S. R., Contreras, S. R., \& López, S. A. (2014). Emprendimiento y MIPYMES (Primera ed.). México: PEARSON.

OIT (2004). Romper el techo de cristal: la mujer en puestos de dirección. Versión actualizada, pp. 32 - 33. Ginebra, Suiza.

Ortiz, C., Duque, Y., Camargo, D. (2008). Una revisión a la investigación en emprendimiento femenino. Revista Facultad de Ciencias Económicas: Investigación y Reflexión, Vol. XVI, No. 1, pp. 85 - 104.

Pereira, L. F. (2007). La evolución del espiritu empresarial como campo del conocimiento: Hacia una visión Sistémica y humanista. Cuaderno de administración, 20(34), 11-37. Recuperado el 12 de 10 de 2013, de http://www.scielo.org.co/ scielo.php?pid=S0120-35922007000200002\&script=sci arttext

Portela, M. M., \& Neira, G. I. (2003). Obtenido de http:// www2.girona.cat/documents/11622/5619109/capital_social.pdf

Porter, M. E. (1980). Ventaja competitiva. México: Grupo Editorial Patria.

Powers, J., \& Magnoni, B. (2010). Dueña de tu propia empresa: Identificación, análisis y superación de las limitaciones a las pequeñas empresas de las mujeres en América Latina y el Caribe. New York: Fondeo multilateral de Inversión Miembros del grupo BID. Obtenido de http://www.mific.gob.ni/Portals/0/Portal\%20Empresarial/Due\%C3\%Bla\%20de\%20 tu\%20propia\%20empresa.pdf

Puchol, L. (2005). El libro del emprendedor como crear tu empresa y convertirte en tu propio jefe (Segunda ed.). Madrid, España: DiazdeSantos.

Quintero, D. C. (2007). Universidad Autónoma de Bucaramanga. Obtenido de Unad. Edu: http://www.usmp.edu.pe/ facarrhh/primer_congreso_ippeu_boletin/ppts/Carlos_ Quinteros.pdf

Ramírez, P. J. (2005). Dimensiones y características del capital social en Guadalajara. México: Universidad Iberoamericana.
Obtenido de http://www.bib.uia.mx/tesis/pdf/014577/0 14577.pdf

Ramírez, R. Y. (2008). «Impacto del Premio Estatal al Emprendedor convocad por el comité Interinstitucional del premio Estatal al emprendedor (CIPEE); en el periodo 1998 -2005, como coadyuvante en la creación y supervivencia de empresas. Aguascalientes: Universidad autónoma de Aguascalientes.

Rodríguez, G. A. (2013). Forbes México. Recuperado el 18 de 06 de 2016, de http://www.forbes.com.mx/el-emprendimiento-femenino/\#gs.hco9Yg0

Rodríguez, R. A. (2009). Nuevas perspectivas para entender el emprendimiento empresarial. (P. M. Dagoberto, Ed.) Pensamiento y gestión, 26, 94-119.

Román, C. R., Gómez, M. A., \& Smida, A. (2013). El Sevier Doyma. Recuperado el 01 de 05 de 2014, de https://www. icesi.edu.co/revistas/index.php/estudios_gerenciales/.../PDF

Sánchez, J. V. (2013). El capital social como instrumento del análisis económico. Cuaderno de Relaciones Laborales, 31(2), 473- 493. Recuperado el 20 de 06 de 2016, de https://revistas.ucm.es/index.php/CRLA/article/viewFile/43227/40977

Sánchez, M. (2010). Mujer y creación de empresas. Tendencias de investigación. (U. d. Extremadura, Ed.) España. Obtenido de http://www.scielo.org.co/scielo.php?script=sci_nlinks \&ref $=000180$ \&pid $=$ S1657-62762013000100009000 $37 \& \operatorname{lng}=\mathrm{en}$

Shaver, K. \& Scott, L. (1991). Person, process, choice: The psychology of new venture creation. https://cemi.com.au/ sites/all/publications/Shaver\%20and\%20Scott\%201991.pdf Surdez, P. E., Aguilar, M. N., \& Rodríguez, M. E. (2008). El empresario de la pequeña empresa. Un Acercamiento a sus características personales. Ponencia VI Congreso Internacional de Análisis Organizacional «Perspectiva multidisciplinaria en análisis organizacional; Complejidad, ambigüedad y subjetividad». Nayarit, México: Universidad Autónoma de Nayarit.

Tambunan, T. (2009). Women entrepreneurships in Asia developing counties: Their development and main constraints. Journal of Development anda Agrucultural Economics, 1(2), 27-40. Obtenido de http://www.smmeresearch.co.za/SMM E\%20Research\%20General/Journal\%20Articles/Women \%20entrepreneurship\%20in\%20Asian\%20developing\%20 countries.pdf 
Vivar, C., Arantzamendi, M. , López-Dicastillo, O., \& Gordo, C. (2010). La Teoría Fundamentada como Metodología de Investigación Cualitativa en Enfermería. Index de Enfermeria, 19(4), 283-288.

Von Neumann, J. \& Morgenstern, O. (1954). Theory of Games and Economic Behavior. Princeton: Princeton University Press. Second edition, 1947; third edition, 1953. Section 3, chapter I. reprinted in Alfred N. Page. (1968). Utility Theory: A Book of Readings. New York: Wiley, pp. 215-233.

Wernerfelt, B. (1984). A resource - based view of the firm. Strategic Management Journal, Vol. 5, No. 2. Pp. $171-180$.
Westerlund, M. \& Svahn, S. (2008). A relationship value perspective of social capital in networks of software SME's, Industrial Marketing Management, 37, 492 - 501.

Zabludovsky, K. G. (2013). Empresarias y Ejecutivas en México Diagnósticos y desafíos (Primera ed.). México: IPADE PUBLISHING.

Zárate, L. M., Vega, C. M., \& Zárate, L. A. (2013). Impacto social de la diversidad de género en instituciones públicas de educación superior en el estado de San Luis Potosí. En M. F. Leal, \& H. J. Ojeda, Desarrollo de la responsabilidad social en las organizaciones. México: Universidad Autónoma de Aguascalientes. 